\title{
Resultate: Der Einfluss des Brand Contents auf das Markenimage
}

Im Folgenden werden die in Kapitel 5 postulierten Hypothesen zur Wirkung von Brand Content auf das Markenimage überprüft. ${ }^{1}$ Das Kapitel ist analog dem Forschungsstand aufgebaut. ${ }^{2}$ Zuerst werden die Haupteffekte von informativem und unterhaltendem Brand Content sowie die Haupteffekte des Kommunikationskanals geprüft. Danach folgen Interaktionseffekte des Kanals, der bestehenden Markenpositionierung und des Produktinvolvements. Der Einfluss der Kontrollvariablen Alter, Geschlecht, Bildung, Einkommen und Affinität zu sozialen Medien wird im Rahmen der Post-hoc-Analysen am Ende des Kapitels geprüft. Einleitend wird das BCBI-Modell auf seine Güte geprüft und die experimentellen Manipulationen werden überprüft.

\footnotetext{
${ }^{1}$ Neben denjenigen Personen, die bereits im Vorwort Erwähnung fanden, sei insbesondere auch Peter Schmidt (Universität Giessen), Eldad Davidod (Universität Köln) und Elmar Schlüter (Universität Giessen) gedankt, die als Dozierende in zwei Kursen (2018 und 2019) über Strukturgleichungsmodelle im Rahmen der «Swiss Summer School in Social Science Methods» wertvolle Tipps und Anregungen zur Auswertung dieser Studie gegeben haben. Ein Dank gebührt schliesslich auch Urs Grob (Universität Zürich) für wertvolle Hinweise zur Auswertung.

${ }^{2}$ Ergänzende Inhalte zu diesem Kapitel finden sich im elektronischen Zusatzmaterial.
}

Elektronisches Zusatzmaterial Die elektronische Version dieses Kapitels enthält Zusatzmaterial, das berechtigten Benutzern zur Verfügung steht https://doi.org/10.1007/978-3-658-35711-5_7. 


\subsection{Brand-Content-Brand-Image(BCBI)-Modell}

Die Messmodelle zum Brand Content, zum Kanal, zum Markenimage und zum markenbezogenen Verhalten (siehe Abschnitt 6.8) und die durch die Hypothesen postulierten Zusammenhänge (Strukturmodell) wurden in einem Strukturgleichungsmodell als Brand-Content-Brand-Image-Modell (BCBI-Modell) abgebildet. Das Modell wurde mit dem bereinigten Datensatz $\left(\mathrm{n}=1^{\prime} 374\right)$ in Mplus 8.0 berechnet. Um auf multivariate Normalverteilung zu prüfen, wurde das Modell sowohl mit ML (Maximum-Likelihood-Schätzung mit konventionellen Standardfehlern) als auch mit MLR (Maximum-Likelihood-Schätzung mit robusten Standardfehlern bei Nicht-Normalverteilung) gerechnet (Muthén \& Muthén, 2017, S. 667). Weil die Güte des Modells mit MLR besser ist, kann nicht von perfekter Normalverteilung ausgegangen werden. Deshalb wird im weiteren Verlauf auf die MLR-Schätzung zurückgegriffen.

In Abschnitt 6.8.6 wurde auf Basis der konfirmatorischen Faktorenanalyse vorgeschlagen, das markenbezogene Verhalten zweizuteilen in eine OnlineDimension und eine «traditionelle» Dimension. Damit ändert sich das durch die Hypothesen abgeleitete Modell. Um zu prüfen, ob diese Aufteilung vorteilhaft ist, wurde zuerst das Ursprungsmodell gerechnet mit einer ungeteilten Variable «markenbezogenes Verhalten». Das Modell verfügt über eine hohe Güte; die Analyse der Modifikationsindizes zeigte jedoch, dass sich insbesondere die Unterhaltungsgratifikation des Brand Contents, die Einstellung gegenüber dem Brand Content und die Informationsgratifikation des Brand Contents auf zwei onlinebezogene Verhaltensvariablen (Online-Verhalten: die Absicht, die Webseite der Marke zu besuchen, und die Absicht, den Brand Content zu teilen) auswirkten. Es wurde ein zweites Modell (BCBI-Modell) berechnet, das, wie in Abschnitt 6.8.6 angedeutet, über zwei unabhängige markenbezogene Verhaltensvariablen verfügt (OnlineVerhalten und traditionelles Verhalten mit Kauf und Weiterempfehlungsabsicht). Zudem wurde ein direkter Pfad von der Einstellung gegenüber dem Brand Content zum Online-Verhalten addiert. Mit einer solchen Modelloptimierung ist ein Strukturgleichungsmodell nicht länger nur konfirmatorischer Natur auf Basis der ursprünglichen Hypothesen, sondern wird zu einem «explorativen Datenanalyseinstrument» (Weiber \& Mühlhaus, 2014, S. 87). Weil das BCBI-Modell auch durch die Modifikationen grösstenteils unverändert bleibt, aber eine bessere Modellgüte aufweist, wird im Folgenden mit dem adaptierten BCBI-Modell gerechnet. Weiter wurden Korrelationen zwischen den Fehlertermen von (a) emotionales Markenimage und funktionales Markenimage, (b) Online-Verhalten und traditionelles Verhalten, (c) wahrgenommene Information und wahrgenommene 
Unterhaltung des Brand Contents und (d) Informationserwartungen und Unterhaltungserwartungen des Kanals zugelassen. Die Korrelation der Fehlertermen latenter Variablen macht Sinn, wenn «gute Gründe» (S. 138) vorliegen, dass die latenten Variablen eine gemeinsame Varianz haben, die nicht durch das Modell erklärt wird (Kline, 2016). Dies ist bei allen genannten Paarungen der Fall. Das adaptierte BCBI-Modell ist in Abbildung. 7.1 wiedergegeben. Das Modell wurde in Mplus (Version 8.0) spezifiziert. Das BCBI-Modell verfügt über eine hohe Güte (siehe Tabelle 7.1). Abgesehen vom $\chi^{2}$-Wert, der, wie in Abschnitt 6.4.3 besprochen, anfällig für die Stichprobengrösse ist, sind alle Güteindikatoren über den erforderlichen Schwellenwerten (Hair et al., 2014, S. 584). Die Korrelationsmatrix inklusive Mittelwerte und Standardabweichungen für alle Indikatoren findet sich im elektronischen Zusatzmaterial (siehe Tabelle B-1).

Tabelle 7.1 Gütemasse des BCBI-Modells

\begin{tabular}{l|l|l|l|l|l|l|l|l}
\hline Name & $d f$ & $\chi^{2}$ & $\chi^{2} / d f$ & $p$ & CFI & TLI & RMSEA & SRMR \\
\hline $\begin{array}{l}\text { Ursprungsmodell } \\
\text { (ML) }\end{array}$ & 535 & 2447.677 & 4.46 & 0.00 & 0.949 & 0.943 & $0.051^{\mathrm{a}}$ & 0.088 \\
\hline $\begin{array}{l}\text { BCBI-Modell } \\
\text { (ML) }\end{array}$ & 533 & 1954.726 & 3.659 & 0.00 & 0.962 & 0.957 & $0.044^{\mathrm{b}}$ & 0.079 \\
\hline $\begin{array}{l}\text { BCBI-Modell } \\
\text { (MLR) }^{\mathrm{d}}\end{array}$ & 533 & 1729.919 & 3.25 & 0.00 & 0.962 & 0.958 & $0.040^{\mathrm{c}}$ & 0.079 \\
\hline
\end{tabular}

Anmerkungen. $\mathrm{ML}=$ Maximum-Likelihood-Schätzung mit konventionellen Standardfehlern, MLR = Maximum-Likelihood-Schätzung mit robusten Standardfehlern gegenüber NichtNormalverteilung (Muthén \& Muthén, 2017, S. 667); CFI = Comparative Fit Index, TLI = Tucker Lewis Fit Index, RMSEA = Root Mean Square Error of Approximation, SRMR = Standardized Root Mean Square Residual.

${ }^{\mathrm{a}}$ K.I. zwischen 0.049 und 0.053 . $^{\mathrm{b}} 90 \%$ K.I. zwischen 0.042 und $0.046{ }^{\mathrm{c}} 90 \%$ K.I. zwischen 0.038 und 0.043 . $^{\mathrm{d}}$ Gewähltes Modell.

Die Determinationskoeffizienten $\mathrm{R}^{2}$ der latenten Variablen sind bis auf eine Ausnahme (Informationserwartungen gegenüber dem Kanal) signifikant. Mit dem BCBI-Modell können die Haupteffekte des Brand Contents und des Kanals geprüft werden. Zur Prüfung der Hypothesen werden jeweils der standardisierte Pfadkoeffizient $(\beta)$ und das mit dem unstandardisierten Pfadkoeffizienten verbundene Signifikanzniveau $(p)$ angegeben. Für Hypothesen, die moderierende Effekte postulieren, wurden Multigruppenanalysen gerechnet. Bei Gruppenvergleichen wird der unstandardisierte Koeffizient verwendet $\left(\beta_{\mathrm{u}}\right)$. 


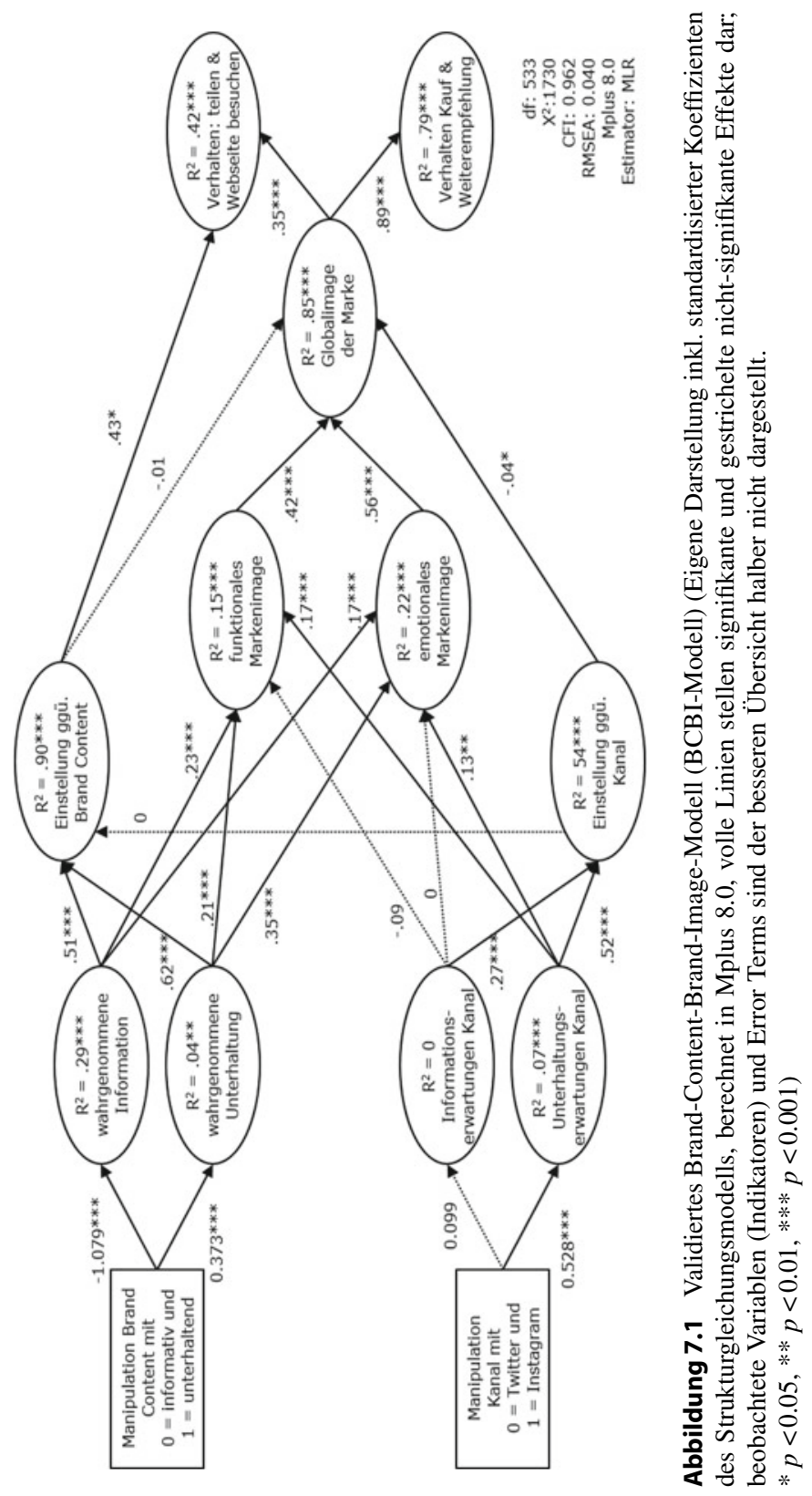




\subsection{Manipulationscheck}

\subsubsection{Brand Content}

Die Manipulation des Brand Contents ${ }^{3}(0=$ informativ, $1=$ unterhaltend $)$ wurde als Dummy-Variable in das BCBI-Modell integriert. Bei dichotomen Prädikatorvariablen muss auf die STDY-Form standardisierter Pfadkoeffizienten zurückgegriffen werden (Muthén \& Muthén, 2017, S. 805). ${ }^{4}$ Die Manipulation wirkt sich gemäss BCBI-Modell signifikant auf die beiden Gratifikationsdimensionen aus (Informationsgratifikation: $\beta=-1.079, p<0.001$; Unterhaltungsgratifikation: $\beta$ $=0.373, p<0.001)$. Der informative Brand Content wird als informativer und weniger unterhaltsam, der unterhaltende Brand Content als weniger informativ und als unterhaltsamer beurteilt. Auf Gesamtebene war die Manipulation entsprechend erfolgreich. Eine Analyse auf der Ebene der Marke zeigt, dass die Manipulation nur bei drei von vier Marken durchgehend gelingt. Bei Rivella bietet der unterhaltsame Brand Content zwar signifikant.weniger Information $(\beta=$ $-0.911, p<0.001)$, aber nicht signifikant mehr Unterhaltung als der informative Brand Content $(\beta=0.204, p=0.073)$.

\subsubsection{Kommunikationskanal}

Die Manipulation des Kanals $(0=$ Twitter [informativ], $1=$ Instagram [unterhaltend]) wirkt sich gemäss BCBI-Modell nur signifikant auf die Unterhaltungsgratifikation aus (Informationsgratifikation: $\beta=0.099, p=0.124$; Unterhaltungsgratifikation: $\beta=0.528, p<0.001) .{ }^{5}$ Die beiden Kanäle unterscheiden sich dementsprechend nicht bezüglich der Informationserwartungen, aber bezüglich der Unterhaltungserwartungen. Obwohl dieser Umstand nicht ideal ist, lässt sich dennoch festhalten, dass Instagram stärker mit Unterhaltung assoziiert wird als Twitter. Inwiefern sich dieser Unterschied auf die Wahrnehmung von

\footnotetext{
${ }^{3}$ Die Manipulation des Brand Contents ist die zentrale Manipulation dieser Arbeit. Im elektronischen Zusatzmaterial in Tabelle B-2 finden sich deshalb die Mittelwerte aller beobachteten Variablen des BCBI-Modells für jeweils beide Ausprägungen des Brand Contents.

${ }^{4}$ Die Werte der STDYX-Standardisierung sind wie folgt: Effekt der Brand-ContentManipulation auf Informationsgratifikation $(\beta=-0.539, p<0.001)$ und auf Unterhaltungsgratifikation $(\beta=0.187, p<0.001)$.

${ }^{5}$ Die Werte der STDYX-Standardisierung sind wie folgt: Effekt der Kanalmanipulation auf Informationserwartungen $(\beta=0.05, p>0.05)$ und Unterhaltungserwartungen $(\beta=0.264, p$ $<0.001)$.
} 
Brand Content auswirkt, soll gemäss den postulierten Hypothesen geprüft werden. Der Einfachheit halber wird im Folgenden Twitter als der (verhältnismässig) informativere und Instagram als der unterhaltende Kanal bezeichnet.

\subsubsection{Markenpositionierung}

Die Manipulation der Markenpositionierung wurde anhand eines einzelnen Items erhoben. Der Manipulationscheck kann über einen Vergleich der Item-Mittelwerte (siehe Tabelle 7.2) mittels einer einfaktoriellen Varianzanalyse (ANOVA) berechnet werden. Weil alle Testpersonen. $\left(n=1^{\prime} 374\right)$ die Markenpositionierung für alle vier Marken eingeschätzt haben, muss eine einfaktorielle Varianzanalyse mit Messwiederholung berechnet werden (Rasch, Friese, Hofmann \& Naumann, 2014b, S. 65, 2014c).

Der Mauchly-Test auf Sphärizität ist signifikant (Mauchly $\mathrm{W}=0.899, d f=$ 5, $p<0.001$ ), was auf eine Verletzung der Annahme der Sphärizität hindeutet. Allerdings neigt der Test bei grossen Stichproben zur Signifikanz. Nichtsdestotrotz wird empfohlen, auf ein Korrekturverfahren zurückzugreifen (Rasch et al., 2014c, S. 4). Sowohl die nach Greenhouse-Geisser korrigierte ( $d f=2.83, \mathrm{~F}=$ 233.417, $p<0.001$ ) als auch die nach Huynh-Feldt korrigierte Berechnung.( $d f$ $=2.84, \mathrm{~F}=233.417, p<0.001)$ deuten auf signifikante Innersubjekteffekte bei der Markenpositionierung hin. Das heisst, dass die einzelnen Teilnehmenden die Markenpositionierung der vier Marken signifikant unterschiedlich beurteilt haben.

Tabelle 7.2 Mittelwerte für die Markenpositionierung der Marken

\begin{tabular}{l|l|l|l}
\hline & $M$ & $S D$ & $n$ \\
\hline Elmex & $2.32^{\mathrm{B}}$ & 1.26 & $950^{\mathrm{a}}$ \\
\hline Rivella & $3.22^{\mathrm{C}}$ & 1.41 & $950^{\mathrm{a}}$ \\
\hline Freitag & $3.09^{\mathrm{C}}$ & 1.35 & $950^{\mathrm{a}}$ \\
\hline V-Zug & $2.12^{\mathrm{A}}$ & 1.11 & $950^{\mathrm{a}}$ \\
\hline
\end{tabular}

Anmerkungen. Berechnet mit SPSS 26 bei listenweiser Löschung. Skala von komplett rationale/kopforientierte Entscheidung, die Marke zu kaufen (1) bis komplett emotionale/gefühlsorientierte Entscheidung, die Marke zu kaufen (5). Signifikante Unterschiede bei den Mittelwerten sind durch unterschiedliche Indizes gekennzeichnet (Bonferroni-Korrektur, $p<0.001)$.

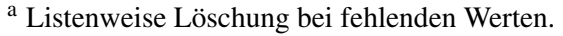


Eine Post-hoc-Analyse mittels paarweiser Vergleiche auf Basis von t-Test und Bonferroni-Korrektur zeigt, dass sich die Mittelwerte von Rivella und Freitag nicht signifikant unterscheiden $\left(M_{R I}-M_{F R}=0.129, p<0.109\right)$, alle anderen Unterschiede jedoch signifikant mit $p<0.001$ sind (siehe elektronisches Zusatzmaterial Tabelle B-3). Obwohl Elmex eine leicht emotionalere Positionierung innehat als V-Zug, werden diese beiden Marken in Übereinstimmung mit der intendierten Manipulation als funktionale Marken zusammengefasst. Rivella und Freitag werden, da beide über eine emotionalere Positionierung als Elmex und V-Zug verfügen, als emotionale Marken betrachtet. Die Manipulation der Markenpositionierung war entsprechend erfolgreich.

\subsubsection{Produktinvolvement}

Die Mittelwerte zum Produktinvolvement der vier Marken wurden mittels Multigruppenanalyse berechnet. Eine CFA über alle latenten Variablen des BCBIModells plus Produktinvolvement zeigt, dass bei den mit den Marken verbundenen Datensätzen keine (partielle) Skalar-Invarianz vorliegt (siehe elektronisches Zusatzmaterial Tabelle B-5). ${ }^{6}$

Wird das Produktinvolvement allein auf Messinvarianz geprüft, liegt ebenfalls weder Skalar-Invarianz noch partielle Skalar-Invarianz vor (siehe elektronisches Zusatzmaterial B-7). Zur Berechnung der partiellen Skalar-Invarianz wurden einmal die Faktorladung und der Achsenabschnitt der Variable PI_2 und einmal der Variable PI_3 frei geschätzt. Beide Lösungen sind ungenügend.

Weil keine partielle Skalar-Invarianz vorliegt, müssen die Mittelwerte mit Vorsicht interpretiert werden. Sie können höchstens als Tendenz verstanden werden. Schliesslich sollen die Unterschiede zwischen den Mittelwerten auch nicht auf Signifikanz geprüft werden (Steinmetz et al., 2009, S. 608). Bei einem Multigruppenvergleich wird der Mittelwerte der ersten Gruppe (hier Elmex) auf 0 gesetzt, während die Mittelwerte weiterer Gruppen als Differenz dazu ausgegeben werden (Tabelle 7.3).

\footnotetext{
${ }^{6}$ Cieciuch und Davidov (2016) empfehlen bei einem Invarianz-Test jeweils zusätzlich die Modellgüte der CFA für sämtliche untersuchten Gruppen anzugeben. Aus Platzgründen werden diese Angaben jeweils im elektronischen Zusatzmaterial aufgeführt. Weil das Produktinvolvement mit drei Indikatoren allerdings gerade identifiziert ist, kann die Modellgüte für eine einzelne Gruppe nicht sinnvoll berechnet werden. Die Angabe entfällt deshalb hier.
} 
Tabelle 7.3 Mittelwerte für das Produktinvolvement der Markenprodukte

\begin{tabular}{l|l|l|l|l}
\hline Marke & $\begin{array}{l}\text { CFA } \\
\text { BCBI-Modell } \\
\text { inkl. PI }\end{array}$ & $\begin{array}{l}\text { CFA BCBI- } \\
\text { Modell inkl. } \\
\text { PI }^{\mathrm{b}}\end{array}$ & CFA PI $^{\mathrm{a}}$ & CFA PI $^{\mathrm{b}}$ \\
\hline $\begin{array}{l}\text { Zahnpasta } \\
\text { (Elmex) }\end{array}$ & 0 & 0 & 0 & 0 \\
\hline $\begin{array}{l}\text { Erfrischungsgetränk } \\
\text { (Rivella) }^{\mathrm{c}}\end{array}$ & -0.106 & -0.389 & -0.246 & -0.383 \\
\hline $\begin{array}{l}\text { Freizeittasche } \\
\text { (Freitag) }^{\mathrm{c}}\end{array}$ & 0.302 & -0.285 & 0.172 & -0.259 \\
\hline $\begin{array}{l}\text { Waschmaschine } \\
\text { (V-Zug) }^{\mathrm{c}}\end{array}$ & 1.276 & 0.809 & 1.215 & 0.811 \\
\hline
\end{tabular}

Anmerkungen . CFA = Confirmatory Factor Analysis; PI = (latente Variable) Produktinvolvement.

a Partielle Skalar-Invarianz, wobei PI_2 über die Gruppen hinweg freigegeben wurde.

b Partielle Skalar-Invarianz, wobei PI_3 über die Gruppen hinweg freigegeben wurde.

${ }^{\mathrm{c}}$ Die Werte der Marken Rivella, Freitag und V-Zug drücken die Differenz zu Elmex aus.

Auf Basis der Mittelwert-Vergleiche ${ }^{7}$ lässt sich feststellen, dass V-Zug (Waschmaschinen) über ein höheres Involvement verfügt als alle anderen Marken. Rivella verfügt über ein tieferes Involvement als alle anderen Marken. Schwierig ist das Produktinvolvement von Freitag einzuordnen, weil es je nachdem, wie das partielle Modell definiert wird, anders hoch ausfällt. Wird die Variable PI_3 über die Gruppen hinweg freigegeben, dann verfügt Freitag über ein tieferes Involvement als Elmex. Wird hingegen die Variable PI_2 über die Gruppen hinweg freigegeben, dann verfügt Freitag über ein höheres Involvement als Elmex. Auf Basis der Ergebnisse lässt sich folgende Tendenz betreffend das Produktinvolvement erkennen: V-Zug > Elmex und Freitag > Rivella.

Es lässt sich damit festhalten, dass die Manipulation des Produktinvolvements nicht wie gewünscht funktioniert hat. Gemäss den Resultaten der Pretests wurde davon ausgegangen, dass V-Zug (Waschmaschinen) und Freitag (Freizeittaschen) über ein hohes Produktinvolvement verfügen, während dieses bei Elmex (Zahnpasta) und Rivella (Süssgetränk) klein ist. Die ungenügende Manipulation des Produktinvolvements hat Auswirkungen auf die Auswertung der Hypothesen zur Markenpositionierung und zum Produktinvolvement. Darauf wird an der entsprechenden Stelle eingegangen.

\footnotetext{
${ }^{7}$ In Tabelle B-4 im elektronischen Zusatzmaterial sind zudem die Mittelwerte der Indikatoren für die vier Marken ausgewiesen.
} 


\subsection{Prüfung der Hypothesen zum Brand Content}

In diesem Kapitel werden mit Ausnahme der kanalbezogenen Hypothesen alle Haupteffekte des BCBI-Modells überprüft.

In einem ersten Schritt wird der Einfluss der Gratifikationen «Information» und «Unterhaltung» geprüft. Es zeigt sich, dass sich sowohl die wahrgenommene Information $(\beta=0.506, p<0.001)$ als auch die wahrgenommene Unterhaltung $(\beta=0.617, p<0.001)$ signifikant positiv auf die Einstellung gegenüber dem Brand Content auswirken. Die Hypothesen 1a und 1b können bestätigt werden (siehe Tabelle 7.4).

Tabelle 7.4 Test der Hypothese 1

\begin{tabular}{l|l|l|l}
\hline $\mathrm{Nr}$ & Hypothese & Koeffizienten & Beurteilung \\
\hline $\mathrm{H} 1$ & $\begin{array}{l}\text { Die wahrgenommene Information (a) und } \\
\text { die wahrgenommene Unterhaltung (b) des }\end{array}$ & $\begin{array}{l}\text { (a) } \beta=0.506, p<0.001 \\
\text { Brand Contents wirken sich positiv auf die }\end{array}$ & bestätigt \\
& $\begin{array}{l}\text { Einstellung gegenüber dem Brand Content } \\
\text { aus }\end{array}$ & & \\
\hline
\end{tabular}

Anmerkungen. $\beta=$ standardisierter Pfadkoeffizient gemäss BCBI-Modell. $p$-Wert auf Basis zweiseitiger Test.

Die Einstellung gegenüber dem Brand Content hat entgegen den Erwartungen keinen signifikanten Einfluss auf das Globalimage $(\beta=-0.012, p=0.481)$. Hypothese 2 wird durch die Daten nicht gestützt (siehe Tabelle 7.5).

Tabelle 7.5 Test der Hypothese 2

\begin{tabular}{l|l|l|l}
\hline $\mathrm{Nr}$ & Hypothese & Koeffizienten & Beurteilung \\
\hline $\mathrm{H} 2$ & $\begin{array}{l}\text { Eine positive Einstellung gegenüber dem } \\
\text { Brand Content wirkt sich positiv auf das } \\
\text { Globalimage der Marke aus }\end{array}$ & $\beta=-0.012, p=0.481$ & abgelehnt \\
\hline
\end{tabular}

Anmerkungen. $\beta=$ standardisierter Pfadkoeffizient gemäss BCBI-Modell. $p$-Wert auf Basis zweiseitiger Test.

Die beiden partiellen Imagedimensionen, das funktionale Markenimage $\left(\beta_{\mathrm{a}}=0.423, p<0.001\right)$ und das emotionale Markenimage $\left(\beta_{\mathrm{b}}=0.557\right.$, $p<0.001$ ), wirken sich in Übereinstimmung mit den Hypothesen 3a und $3 \mathrm{~b}$ beide signifikant auf das Globalimage aus (siehe Tabelle 7.6). Ein Vergleich der beiden unstandardisierten Parameter mittels eines $\chi^{2}$-Differenz-Tests 
(Muthén \& Muthén, 2017, S. 772) offenbart, dass sich diese in ihrer Stärke nicht unterscheiden $\left(\beta_{\mathrm{ua}}-\beta_{\mathrm{ub}}=0.061, p=0.569\right)$.

Tabelle 7.6 Test der Hypothese 3

\begin{tabular}{l|l|l|l}
\hline $\mathrm{Nr}$ & Hypothese & Koeffizienten & Beurteilung \\
\hline$H 3$ & $\begin{array}{l}\text { Das funktionale (a) und das emotionale } \\
\text { Markenimage (b) wirken sich positiv auf das } \\
\text { Globalimage der Marke aus }\end{array}$ & $\begin{array}{l}\text { (a) } \beta=0.423, p<0.001 \\
\text { (b) } \beta=0.557, p<0.001\end{array}$ & bestätigt \\
\hline
\end{tabular}

Anmerkungen. $\beta=$ standardisierter Pfadkoeffizient gemäss BCBI-Modell. $p$-Wert auf Basis zweiseitiger Test.

Im Folgenden wird der sogenannte «Innuendo-Effekt» überprüft. Dieser besagt, dass eine Wahrnehmungskomponente B negativ beurteilt wird, wenn nur eine andere Wahrnehmungskomponente A positiv beschrieben wird (Kervyn et al., 2012). Die Auswertung zeigt, dass sich die wahrgenommene Information sowohl positiv auf das funktionale Markenimage $(\beta=0.225, p<0.001)$ als auch positiv auf das emotionale Markenimage $(\beta=0.166, p<0.001)$ auswirkt. Der Unterschied zwischen den beiden (unstandardisierten) Parametern ist nicht signifikant. $\left(\beta_{\mathrm{ua}}-\beta_{\mathrm{ub}}=-0.013, p=0.59\right)$, wie ein $\chi^{2}$-Differenz-Test zeigt. Es lässt sich damit festhalten, dass das funktionale und das emotionale Markenimage gleichermassen positiv durch die wahrgenommene Information des Brand Contents profitieren. Damit ist Hypothese $4 \mathrm{a} \mathrm{zu}$ bestätigen und $4 \mathrm{~b}$ abzulehnen (siehe Tabelle 7.7). Ein Innuendo-Effekt kann entsprechend nicht nachgewiesen werden.

Tabelle 7.7 Test der Hypothese 4

\begin{tabular}{l|l|l|l}
\hline $\mathrm{Nr}$ & Hypothese & Koeffizienten & Beurteilung \\
\hline$H 4$ & $\begin{array}{l}\text { Die wahrgenommene Information des } \\
\text { Brand Contents hat einen positiven Einfluss } \\
\text { auf die funktionale Dimension (a) des } \\
\begin{array}{l}\text { Markenimages und einen negativen Effekt } \\
\text { auf die emotionale Dimension (b) des } \\
\text { Markenimages }\end{array}\end{array}$ & $\begin{array}{l}\text { (a) } \beta=0.225, p<0.001 \\
\text { (b) } \beta=0.166, p<0.001\end{array}$ & $\begin{array}{l}\text { a: bestätigt } \\
\text { b: abgelehnt }\end{array}$ \\
\hline
\end{tabular}

Anmerkungen. $\beta=$ standardisierter Pfadkoeffizient gemäss BCBI-Modell. $p$-Wert auf Basis zweiseitiger Test. 
Auch bezüglich Unterhaltung kann kein solcher Effekt nachgewiesen werden. Die wahrgenommene Unterhaltung des Brand Contents beeinflusst sowohl signifikant positiv das emotionale Markenimage $(\beta=0.353, p<0.001)$ als auch signifikant positiv das funktionale Markenimage $(\beta=0.213, p<0.001)$. Der Effekt der Unterhaltung auf das emotionale Markenimage ist signifikant stärker als der Effekt auf das funktionale Markenimage, wie eine Gegenüberstellung der unstandardisierten Parameter auf Basis eines $\chi^{2}$-Differenz-Tests zeigt $\left(\beta_{\mathrm{ua}}-\beta_{\mathrm{ub}}\right.$ $=0.210, p<0.001)$. Auch hier kann Hypothese 5a bestätigt werden, während Hypothese $5 \mathrm{~b}$ zurückgewiesen werden muss (siehe Tabelle 7.8).

Tabelle 7.8 Test der Hypothese 5

\begin{tabular}{l|l|l|l}
\hline $\mathrm{Nr}$ & Hypothese & Koeffizienten & Beurteilung \\
\hline$H 5$ & $\begin{array}{l}\text { Die wahrgenommene Unterhaltung des } \\
\text { Brand Contents hat einen positiven Einfluss } \\
\text { auf die emotionale Dimension (a) des } \\
\text { Markenimages und einen negativen Effekt } \\
\text { auf die funktionale Dimension (b) des } \\
\text { Markenimages }\end{array}$ & $\begin{array}{l}\text { (a) } \beta=0.353, p<0.001 \\
\text { (b) } \beta=0.213, p<0.001\end{array}$ & $\begin{array}{l}\text { a: bestätigt } \\
\text { b: abgelehnt }\end{array}$ \\
\hline
\end{tabular}

Anmerkungen. $\beta=$ standardisierter Pfadkoeffizient gemäss BCBI-Modell. $p$-Wert auf Basis zweiseitiger Test.

Vergleicht man, wie stark die Partialimages durch die beiden Gratifikationen determiniert werden, können bezüglich des funktionalen Markenimages keine signifikanten Unterschiede zwischen Information und Unterhaltung ausgemacht werden $\left(\beta_{\mathrm{uI}}-\beta_{\mathrm{uU}}=-0.03, p=0.937\right)$. Das emotionale Markenimage wird allerdings signifikant stärker durch Unterhaltung als durch Information beeinflusst $\left(\beta_{\mathrm{uU}}-\beta_{\mathrm{uI}}=0.201, p<0.001\right)$.

Bei den Hypothesen 6 und 7 handelt es sich um einen postulierten Interaktionseffekt mit einer latenten Variablen als Moderator. Analog der klassischen Berechnung von Interaktionen wird hierzu eine Interaktionsvariable gebildet durch die Multiplikation von Information bzw. Unterhaltung mit der Einstellung gegenüber dem Brand Content (Urban \& Mayerl, 2014, S. 41). Die abhängige Variable (funktionales bzw. emotionales Markenimage) wird zusätzlich auf die Interaktionsvariable $\left(\beta_{\text {Int) }}\right.$ und die Variable «Einstellung gegenüber dem Brand Content» regrediert. Um die Interaktionsvariable und deren Effekt zu berechnen, gibt es für SEM verschiedene Vorgehensweisen (Kline, 2016, S. 437; Steinmetz, Davidov \& Schmidt, 2011). Mplus wendet das LMS-Verfahren (Latent Moderated Structural Equations) nach A. Klein und Moosbrugger (2000) an (Muthén \& Muthén, 2017, S. 79). 
Die Auswertung zeigt, dass die Einstellung gegenüber dem Brand Content weder die Wirkung der Information auf das funktionale Markenimage $\left(\beta_{\text {Int }}=\right.$ $0.005, p=0.850$ ) noch die Wirkung der Unterhaltung auf das emotionale Markenimage $\left(\beta_{\text {Int }}=0.018, p=0.384\right)$ moderiert. Die beiden Hypothesen 6 und 7 müssen entsprechend abgelehnt werden (siehe Tabelle 7.9 und.Tabelle 7.10).

Tabelle 7.9 Test der Hypothese 6

\begin{tabular}{l|l|l|l}
\hline $\mathrm{Nr}$ & Hypothese & Koeffizienten & Beurteilung \\
\hline$H 6$ & $\begin{array}{l}\text { Der Einfluss der wahrgenommenen } \\
\text { Information des Brand Contents auf } \\
\text { das funktionale Markenimage ist } \\
\text { stärker bei positiver (im Vergleich zu } \\
\text { negativer) Einstellung gegenüber dem } \\
\text { Brand Content }\end{array}$ & $\beta_{\text {Int }}=0.005, p=0.850$ & abgelehnt \\
\hline
\end{tabular}

Anmerkungen. $\beta_{\text {Int }}=$ standardisierter Pfadkoeffizient der Interaktionsvariable. $p$-Wert auf Basis zweiseitiger Test.

Tabelle 7.10 Test der Hypothese 7

\begin{tabular}{l|l|l|l}
\hline $\mathrm{Nr}$ & Hypothese & Koeffizienten & Beurteilung \\
\hline$H 7$ & $\begin{array}{l}\text { Der Einfluss der wahrgenommenen } \\
\text { Unterhaltung des Brand Contents auf } \\
\text { das emotionale Markenimage ist } \\
\text { stärker bei positiver (im Vergleich zu } \\
\text { negativer) Einstellung gegenüber dem } \\
\text { Brand Content }\end{array}$ & $\beta_{\text {Int }}=0.018, p=0.384$ & abgelehnt \\
\hline
\end{tabular}

Anmerkungen. $\beta_{\text {Int }}=$ standardisierter Pfadkoeffizient der Interaktionsvariable. $p$-Wert auf Basis zweiseitiger Test.

Schliesslich wird im Modell geprüft, inwiefern das Globalimage das markenbezogene Verhalten beeinflusst. Es wurde eingangs dieses Kapitels bei der Spezifikation des BCBI-Modells aufgezeigt, dass das markenbezogene Verhalten in zwei latente Variablen aufgeteilt wird. Im Online-Verhalten geht es darum, Brand Content zu teilen oder die Webseite der Marke zu besuchen. Das traditionelle Verhalten umfasst die Kaufabsicht und die Einschätzung, inwiefern man die Marke weiterempfehlen würde. Die Auswertungen zeigen, dass das Globalimage sowohl das Online-Verhalten $(\beta=0.347, p<0.001)$ als auch das traditionelle markenbezogene Verhalten $(\beta=0.890, p<0.001)$ positiv beeinflusst. Dadurch 
werden die Hypothesen 8a und 8b durch die Daten gestützt (siehe Tabelle 7.11 und Tabelle 7.12).

Tabelle 7.11 Test der Hypothese 8a

\begin{tabular}{l|l|l|l}
\hline $\mathrm{Nr}$ & Hypothese & Koeffizienten & Beurteilung \\
\hline $\mathrm{H} 8 \mathrm{a}$ & $\begin{array}{l}\text { Das Globalimage der Marke wirkt sich positiv } \\
\text { auf das markenbezogene Online-Verhalten aus }\end{array}$ & $\beta=0.347, p<0.001$ & bestätigt \\
\hline
\end{tabular}

Anmerkungen. $\beta=$ standardisierter Pfadkoeffizient gemäss BCBI-Modell. $p$-Wert auf Basis zweiseitiger Test.

Tabelle 7.12 Test der Hypothese 8b

\begin{tabular}{l|l|l|l}
\hline $\mathrm{Nr}$ & Hypothese & Koeffizienten & Beurteilung \\
\hline$H 8 b$ & $\begin{array}{l}\text { Das Globalimage der Marke wirkt sich positiv } \\
\text { auf das traditionelle markenbezogene } \\
\text { Verhalten aus }\end{array}$ & $\beta=0.890, p<0.001$ & bestätigt \\
\hline
\end{tabular}

Anmerkungen. $\beta=$ standardisierter Pfadkoeffizient gemäss BCBI-Modell. $p$-Wert auf Basis zweiseitiger Test.

Weiter zeigt sich, dass das Online-Verhalten signifikant positiv durch die Einstellung gegenüber dem Brand Content beeinflusst wird $(\beta=0.433, p$ $<0.001)$.

\subsection{Prüfung der Hypothesen zum Kommunikationskanal}

In diesem Kapitel werden die kanalbezogenen Hypothesen geprüft. Auch hier folgt der Aufbau dem Kapitel Forschungsstand. Zuerst werden die Haupteffekte des Kanals gemäss BCBI- Modell geprüft, danach folgen Interaktionseffekte des Kanals.

Als Erstes wird der Einfluss der Informations- und Unterhaltungserwartungen auf die Einstellung gegenüber dem Kanal geprüft. Es zeigt sich, dass die Einstellung des Kanals in Übereinstimmung mit Hypothese 9 sowohl von den Informationserwartungen $(\beta=0.269, p<0.001)$ als auch von den Unterhaltungserwartungen $(\beta=0.515, p<0.001)$ positiv beeinflusst wird (siehe Tabelle 7.13). 
Tabelle 7.13 Test der Hypothese 9

\begin{tabular}{l|l|l|l}
\hline $\mathrm{Nr}$ & Hypothese & Koeffizienten & Beurteilung \\
\hline$H 9$ & $\begin{array}{l}\text { Die Informations- (a) und } \\
\text { Unterhaltungserwartungen (b) gegenüber } \\
\text { dem digitalen Kommunikationskanals } \\
\text { wirken sich positiv auf die Einstellung } \\
\text { gegenüber dem Kanal aus }\end{array}$ & $\begin{array}{l}\text { (a) } \beta=0.269, p<0.001 \\
\text { (b) } \beta=0.515, p<0.001\end{array}$ & bestätigt \\
\hline
\end{tabular}

Anmerkungen. $\beta=$ standardisierter Pfadkoeffizient gemäss BCBI-Modell. $p$-Wert auf Basis zweiseitiger Test.

Entgegen Hypothese 10 kann kein signifikanter Effekt der positiven Einstellung gegenüber dem Kanal auf die Einstellung gegenüber dem Brand Content festgestellt werden ( $\beta=-0.01, p=0.932$; siehe Tabelle 7.14).

Tabelle 7.14 Test der Hypothese 10

\begin{tabular}{l|l|l|l}
\hline $\mathrm{Nr}$ & Hypothese & Koeffizienten & Beurteilung \\
\hline$H 10$ & $\begin{array}{l}\text { Eine positive Einstellung gegenüber dem } \\
\text { Kanal wirkt sich positiv auf die Einstellung } \\
\text { gegenüber dem Brand Content aus }\end{array}$ & $\beta=-0.001, p=0.932$ & abgelehnt \\
\hline
\end{tabular}

Anmerkungen. $\beta=$ standardisierter Pfadkoeffizient gemäss BCBI-Modell. $p$-Wert auf Basis zweiseitiger Test.

Für den Einfluss der Einstellung gegenüber dem Kanal auf das Globalimage der Marke kann ein signifikant negativer Einfluss auf dem $5 \%$-Niveau festgestellt werden $(\beta=-0.04, p=0.021)$. Ein negativer Einfluss steht dem in der Hypothese postuliertem Effekt konträr entgegen, womit auf Basis dieser Studie Hypothese 11 nicht bestätigt werden kann (siehe Tabelle 7.15). Allerdings ist dieser Effekt marginal und deutlich unter der Schwelle von $\beta=0.2$, ab welcher von einem bedeutungsvollen Zusammenhang gesprochen werden kann (Chin, 1998).

Mit den beiden nächsten Hypothesen wird getestet, ob sich die mit dem Kanal verbundenen Gratifikationserwartungen auf das funktionale oder emotionale Markenimage übertragen. Hypothese 12 untersucht dazu den Einfluss der Informationserwartungen gegenüber dem Kanal. Es zeigt sich anhand der berechneten Koeffizienten, dass die Informationserwartungen keinen signifikanten Einfluss auf die funktionale $\left(\beta_{\mathrm{a}}=-0.085, p=0.083\right)$ oder auf die emotionale Dimension $\left(\beta_{\mathrm{b}}=0.003, p=0\right.$. 956) des Markenimages haben. Entsprechend 
Tabelle 7.15 Test der Hypothese 11

\begin{tabular}{l|l|l|l}
\hline $\mathrm{Nr}$ & Hypothese & Koeffizienten & Beurteilung \\
\hline$H 11$ & $\begin{array}{l}\text { Eine positive Einstellung gegenüber } \\
\text { dem Kanal wirkt sich positiv auf das } \\
\text { Globalimage der Marke aus }\end{array}$ & $\beta=-0.040, p=0.021$ & abgelehnt \\
\hline
\end{tabular}

Anmerkungen. $\beta=$ standardisierter Pfadkoeffizient gemäss BCBI-Modell. $p$-Wert auf Basis zweiseitiger Test.

ist Hypothese 12 abzulehnen (siehe Tabelle 7.16). Weil sich die beiden Koeffizienten nicht signifikant von 0 unterscheiden, unterscheiden sie sich auch nicht voneinander. Ein Vergleich der unstandardisierten Parameter anhand eines $\chi^{2}$-Differenz-Tests bestätigt dies $\left(\beta_{\mathrm{ua}}-\beta_{\mathrm{ub}}=-0.059, p=0.145\right)$.

Tabelle 7.16 Test der Hypothese 12

\begin{tabular}{l|l|l|l}
\hline $\mathrm{Nr}$ & Hypothese & Koeffizienten & Beurteilung \\
\hline$H 12$ & $\begin{array}{l}\text { Die Informationserwartungen } \\
\text { gegenüber dem Kanal haben einen } \\
\text { stärkeren positiven Einfluss auf } \\
\text { die funktionale Dimension (a) des } \\
\text { Markenimages als auf die } \\
\text { emotionale Dimension (b) }\end{array}$ & $\begin{array}{l}\text { (a) } \beta=-0.085, p=0.083 \\
\text { (b) } \beta=0.003, p=0.956\end{array}$ & abgelehnt \\
\hline
\end{tabular}

Anmerkungen. $\beta=$ standardisierter Pfadkoeffizient gemäss BCBI-Modell. $p$-Wert auf Basis zweiseitiger Test.

Analog dazu postuliert Hypothese 13 einen Effekt der Unterhaltungserwartungen gegenüber dem Kanal auf das funktionale und das emotionale Markenimage. Im Gegensatz zu den Informationserwartungen kann ein signifikanter Effekt der Unterhaltungserwartungen sowohl auf das emotionale Markenimage $\left(\beta_{\mathrm{a}}=0.133\right.$, $p=0.006)$ als auch auf das funktionale Markenimage $\left(\beta_{\mathrm{b}}=0.171, p<0.001\right)$ nachgewiesen werden. Ein Vergleich der unstandardisierten Koeffizienten offenbart allerdings, dass sich die beiden Effekte nicht signifikant unterscheiden ( $\beta_{\text {ua }}$ $\left.-\beta_{\mathrm{ub}}=-0.025, p=0.619\right)$. Es lässt sich festhalten, dass die Unterhaltungsgratifikation des Kanals beide Partialimages der Marke positiv beeinflusst. Demnach ist Hypothese 13 zurückzuweisen (siehe Tabelle 7.17).

Bei den beiden folgenden Hypothesen handelt es sich um Interaktionseffekte, die besagen, dass die Wirkung des informativen und unterhaltenden Brand Contents von den mit dem Kanal assoziierten Gratifikationserwartungen abhängt. Um 
Tabelle 7.17 Test der Hypothese 13

\begin{tabular}{l|l|l|l}
\hline $\mathrm{Nr}$ & Hypothese & Koeffizienten & Beurteilung \\
\hline$H 13$ & $\begin{array}{l}\text { Die Unterhaltungserwartungen } \\
\text { gegenüber dem Kanal haben einen } \\
\text { stärkeren positiven Einfluss auf die } \\
\text { emotionale Dimension (a) des } \\
\text { Markenimages als auf die } \\
\text { funktionale Dimension (b) }\end{array}$ & $\begin{array}{l}\text { (a): } \beta=0.133, p=0.006 \\
\text { (b): } \beta=0.171, p<0.001\end{array}$ & abgelehnt \\
\hline
\end{tabular}

Anmerkungen. $\beta=$ standardisierter Pfadkoeffizient gemäss BCBI-Modell. $p$-Wert auf Basis zweiseitiger Test.

die beiden Hypothesen zum Ausmass der Einstellung gegenüber dem Brand Content und zum Globalimage der Marke zu testen, wurde der Datensatz in vier Subsamples unterteilt, je nach Manipulation von Brand Content und Kanal (siehe etwa K.-J. Chen et al., 2015; Dahlén et al., 2008; Ryffel et al., 2014):

- Sample 1 (IBC_TW) mit $n=342$ : Informativer Brand Content $\&$ informativer Kanal (Twitter)

- Sample 2 (IBC_IN) mit $n=355$ : Informativer Brand Content $\&$ unterhaltsamer Kanal (Instagram)

- Sample 3 (UBC_TW) mit $n=333$ : Unterhaltender Brand Content \& informativer Kanal (Twitter)

- Sample 4 (UBC_IN) mit $n=344$ : Unterhaltender Brand Content \& unterhaltender Kanal (Instagram)

Den Hypothesen nach müssten die Mittelwerte zur Einstellung gegenüber dem Brand Content und zum Globalimage der Marke in Sample 1 höher sein als in Sample 2 (Hypothese 14) und in Sample 4 höher als in Sample 3 (Hypothese 15). Mittelwerte können mittels Structured Means Modeling (Breitsohl, 2019) verglichen werden (siehe Abschnitt 6.4.6). Dazu müssen die verschiedenen Samples auf Messvarianz getestet werden. Nur wenn die Messmodelle zu den latenten Variablen in allen Samples gleich sind, ist der Vergleich der Mittelwerte zulässig (Cieciuch \& Davidov, 2016). Um die vier Gruppen auf Messinvarianz zu testen, wurde eine Multigruppen-CFA über alle latenten Variablen des Modells (GesamtCFA) gerechnet. Die Resultate zeigen, dass die Modellgüte mit zunehmenden Restriktionen nicht schlechter wird und Messvarianz entsprechend vorliegt (siehe elektronisches Zusatzmaterial Tabelle B-9; F. F. Chen, 2007). Zusätzlich wurde getestet, ob Messinvarianz vorliegt, wenn nur die abhängigen Variablen «Einstellung gegenüber dem Brand Content» und «Globalimage der Marke» geprüft 
wurden. Auch in diesem reduzierten Modell konnte Invarianz festgestellt werden (siehe elektronisches Zusatzmaterial Tabelle B-11). Die folgenden Angaben beziehen sich auf die Gesamt-CFA.

Der Mittelwert der Einstellung gegenüber dem informativen Brand Content ist nicht signifikant höher, wenn dieser im informativen Kanal $\left(M_{T W}\right.$; Twitter) anstatt im unterhaltenden Kanal. ( $M_{I N}$; Instagram) gezeigt wird $\left(M_{T W}-M_{I N}=\right.$ $-0.012, p=0.878$ ). Auch das Globalimage der Marke wird nicht signifikant besser beurteilt, wenn der informative Brand Content über Twitter angeschaut wird $\left(M_{T W}-M_{I N}=-0.126, p=0.091\right)$. Die Hypothesen $14 \mathrm{a}$ und $14 \mathrm{~b}$ müssen entsprechend abgelehnt werden (siehe Tabelle 7.18 und Tabelle 7.19).

Tabelle 7.18 Test der Hypothese 14a

\begin{tabular}{l|l|l|l}
\hline $\mathrm{Nr}$ & Hypothese & Koeffizienten & Beurteilung \\
\hline $\mathrm{H} 14 a$ & $\begin{array}{l}\text { Die Einstellung gegenüber dem } \\
\text { Brand Content ist höher, wenn } \\
\text { informativer Brand Content in einem } \\
\text { informativen (anstatt unterhaltenden) } \\
\text { Kanal gezeigt wird }\end{array}$ & $\begin{array}{l}M_{T W}-M_{I N}=-0.012, \\
p=0.878\end{array}$ & abgelehnt \\
\hline
\end{tabular}

Anmerkungen. $M_{T W}=$ Mittelwert für «Einstellung gegenüber (informativem) Brand Content» im informativen Kanal (Twitter). $M_{I N}=$ Mittelwert für «Einstellung gegenüber (informativem) Brand Content» im unterhaltenden Kanal (Instagram). Berechnung erfolgte mittels SMM auf Basis einer Gesamt-CFA (alle LVs des BCBI-Modells). p-Wert auf Basis zweiseitiger Test.

Tabelle 7.19 Test der Hypothese 14b

\begin{tabular}{l|l|l|l}
\hline $\mathrm{Nr}$ & Hypothese & Koeffizienten & Beurteilung \\
\hline$H 14 b$ & $\begin{array}{l}\text { Das Globalimage der Marke ist } \\
\text { höher, wenn informativer Brand } \\
\text { Content in einem informativen } \\
\text { (anstatt unterhaltenden) Kanal } \\
\text { gezeigt wird }\end{array}$ & $\begin{array}{l}M_{T W}-M_{I N}=-0.126, \\
p=0.091\end{array}$ & abgelehnt \\
\hline
\end{tabular}

Anmerkungen. $M_{T W}=$ Mittelwert für «Globalimage» bei informativen Brand Content im informativen Kanal (Twitter). $M_{I N}=$ Mittelwert für «Globalimage» bei informativen Brand Content im unterhaltenden Kanal (Instagram). Berechnung erfolgte mittels SMM auf Basis einer Gesamt-CFA (alle LVs des BCBI-Modells). p-Wert auf Basis zweiseitiger Test.

Für den unterhaltenden Brand Content ergaben sich folgende Interaktionseffekte des Kanals: Weder die Einstellung gegenüber dem unterhaltenden Brand 
Content $\left(M_{I N}-M_{T W}=-0.047, p=0.590\right)$ noch das Globalimage $\left(M_{I N}-M_{T W}\right.$ $=0.096, p=0.230$ ) unterscheiden sich, je nachdem, ob der unterhaltende Brand Content auf Instagram $\left(M_{I N}\right)$ oder auf Twitter $\left(M_{T W}\right)$ angesehen wurde. Entsprechend sind auch die Hypothesen 15a und 15b abzulehnen (siehe Tabelle 7.20 und Tabelle 7.21).

Tabelle 7.20 Test der Hypothese 15a

\begin{tabular}{l|l|l|l}
\hline $\mathrm{Nr}$ & Hypothese & Koeffizienten & Beurteilung \\
\hline $\mathrm{H} 15 \mathrm{a}$ & $\begin{array}{l}\text { Die Einstellung gegenüber dem } \\
\text { Brand Content ist höher, wenn } \\
\text { unterhaltender Brand Content in } \\
\text { einem unterhaltenden (anstatt } \\
\text { informativen) Kanal gezeigt wird }\end{array}$ & $\begin{array}{l}M_{I N}-M_{T W}=-0.047, \\
p=0.590\end{array}$ & abgelehnt \\
\end{tabular}

Anmerkungen. $M_{I N}=$ Mittelwert für «Einstellung gegenüber (unterhaltendem) Brand Content» im unterhaltenden Kanal (Instagram). $M_{T W}=$ Mittelwert für «Einstellung gegenüber (unterhaltendem) Brand Content» im informativen Kanal (Twitter). Berechnung erfolgte mittels SMM auf Basis einer Gesamt-CFA (alle LVs des BCBI-Modells). p-Wert auf Basis zweiseitiger Test.

Tabelle 7.21 Test der Hypothese 15b

\begin{tabular}{l|l|l|l}
\hline $\mathrm{Nr}$ & Hypothese & Koeffizienten & Beurteilung \\
\hline$H 15 b$ & $\begin{array}{l}\text { Das Globalimage der Marke ist höher, wenn } \\
\text { unterhaltender Brand Content in einem } \\
\text { unterhaltenden (anstatt informativen) Kanal } \\
\text { gezeigt wird }\end{array}$ & $\begin{array}{l}M_{I N}-M_{T W}=0.096, \\
p=0.230\end{array}$ & abgelehnt \\
\hline
\end{tabular}

Anmerkungen. $M_{I N}=$ Mittelwert für «Globalimage» bei unterhaltendem Brand Content im unterhaltenden Kanal (Instagram). $M_{T W}=$ Mittelwert für «Globalimage» bei unterhaltendem Brand Content im informativen Kanal (Twitter). Berechnung erfolgte mittels SMM auf Basis einer Gesamt-CFA (alle LVs des BCBI-Modells). p-Wert auf Basis zweiseitiger Test.

Auf Basis dieser Resultate kann kein Interaktionseffekt des Kanals auf die Wirkung von Brand Content festgestellt werden. 


\subsection{Prüfung der Hypothesen zur Markenpositionierung und zum Produktinvolvement}

Unter Abschnitt 7.2 wurde aufgezeigt, dass die Manipulation der Markenpositionierung erfolgreich ist. Elmex und V-Zug können als funktionale, Rivella und Freitag als emotionale Marken zusammengefasst werden. Allerdings war die Manipulation des Produktinvolvements nicht gelungen. V-Zug verfügt über das höchste Produktinvolvement, Freitag und Elmex über ein mittleres und Rivella über ein tiefes Produktinvolvement.

Die unzulängliche Manipulation des Involvements erschwert nicht nur die Analyse der Hypothesen zum Produktinvolvement, sondern hat auch Konsequenzen auf die Überprüfung der Hypothesen zur Markenpositionierung. Die beiden funktionalen Marken (V-Zug und Elmex, $n=688$ ) verfügen damit über ein höheres Produktinvolvement als die beiden emotionalen Marken (Freitag und Rivella, $n=686$ ).

Durch das höhere Produktinvolvement der funktionalen Marken ergibt sich eine Konfundierung der unabhängigen Variablen. Unterschiede zwischen funktionalen und emotionalen Marken können sich sowohl durch die Markenpositionierung als auch durch das Involvement ergeben. Aus diesem Grund werden im Folgenden die vier Marken direkt miteinander verglichen. Es soll damit geprüft werden, ob sich die postulierten Effekte nach den Marken unterscheiden. Dieses Vorgehen ermöglicht einen indirekten Einblick in die Wirkung von Markenpositionierung und Produktinvolvement. Dafür müssen die postulierten Hypothesen zur Markenpositionierung und zum Produktinvolvement den neuen Gegebenheiten angepasst werden (siehe Tabelle 7.22).

Zuerst wird geprüft, ob die Messmodelle über die vier Marken hinweg invariant sind. Bei einer gemeinsamen CFA über alle latenten Variablen des BCBI-Modells (Gesamt-CFA) verschlechtert sich die Modellgüte mit zunehmenden Restriktionen nicht signifikant (siehe elektronisches Zusatzmaterial Tabelle B-13; F. F. Chen, 2007). Deshalb kann festgehalten werden, dass Messinvarianz über die vier Marken vorliegt.

Zur Prüfung der ersten beiden Hypothesen wird betrachtet, inwiefern informativer im Vergleich zu unterhaltendem Brand Content pro Marke wirkt. Den ursprünglichen Hypothesen nach sollten die Einstellung gegenüber dem Brand Content und das Globalimage der Marke bei V-Zug (funktionales High Involvement) am höchsten bei informativem Brand Content ausfallen. Bei Rivella (emotionales Low Involvement) sollten die beiden abhängigen Variablen am höchsten bei unterhaltendem Brand Content sein. 
Tabelle 7.22 Umformulierung der Hypothesen

\begin{tabular}{|c|c|c|}
\hline $\begin{array}{l}\text { Hypothese zur } \\
\text { Markenpositionierung (alt) }\end{array}$ & $\begin{array}{l}\text { Hypothese zum } \\
\text { Produktinvolvement (alt) }\end{array}$ & $\begin{array}{l}\text { Hypothese zu den Marken } \\
\text { (neu) }\end{array}$ \\
\hline $\begin{array}{l}\text { H16: Die Einstellung } \\
\text { gegenüber dem Brand } \\
\text { Content (a) und das } \\
\text { Globalimage der Marke (b) } \\
\text { sind höher, wenn eine } \\
\text { funktionale Marke mit } \\
\text { informativem (anstatt } \\
\text { unterhaltendem) Brand } \\
\text { Content kommuniziert }\end{array}$ & $\begin{array}{l}\text { H22: Die Einstellung } \\
\text { gegenüber dem Brand } \\
\text { Content (a) und das } \\
\text { Globalimage der Marke (b) } \\
\text { sind höher, wenn eine } \\
\text { High-Involvement-Marke } \\
\text { mit informativem (anstatt } \\
\text { unterhaltendem) Brand } \\
\text { Content kommuniziert }\end{array}$ & \multirow[t]{2}{*}{$\begin{array}{l}\text { H16_N: Es gibt einen } \\
\text { Interaktionseffekt zwischen } \\
\text { Marke und Brand Content } \\
\text { betreffend die Einstellung } \\
\text { gegenüber dem Brand } \\
\text { Content (a) und das } \\
\text { Globalimage (b) }\end{array}$} \\
\hline $\begin{array}{l}\text { H17: Die Einstellung } \\
\text { gegenüber dem Brand } \\
\text { Content (a) und das } \\
\text { Globalimage der Marke (b) } \\
\text { sind höher, wenn eine } \\
\text { emotionale Marke mit } \\
\text { unterhaltendem (anstatt } \\
\text { informativem) Brand } \\
\text { Content kommuniziert }\end{array}$ & $\begin{array}{l}\text { H23: Die Einstellung } \\
\text { gegenüber dem Brand } \\
\text { Content (a) und das } \\
\text { Globalimage der Marke (b) } \\
\text { sind höher, wenn eine } \\
\text { Low-Involvement-Marke mit } \\
\text { unterhaltendem (anstatt } \\
\text { informativem) Brand } \\
\text { Content kommuniziert }\end{array}$ & \\
\hline $\begin{array}{l}\text { H18: Der Einfluss der } \\
\text { wahrgenommenen } \\
\text { Information des Brand } \\
\text { Contents auf das funktionale } \\
\text { Markenimage ist stärker für } \\
\text { emotionale (im Vergleich zu } \\
\text { funktionalen) Marken }\end{array}$ & $\begin{array}{l}\text { H24: Der Einfluss der } \\
\text { wahrgenommenen } \\
\text { Information des Brand } \\
\text { Contents auf das funktionale } \\
\text { Markenimage ist stärker für } \\
\text { High-Involvement-Marken } \\
\text { im Vergleich zu } \\
\text { Low-Involvement-Marken }\end{array}$ & $\begin{array}{l}\text { H18_N: Der Einfluss der } \\
\text { wahrgenommenen } \\
\text { Information des Brand } \\
\text { Contents auf das funktionale } \\
\text { Markenimage wird durch die } \\
\text { Marke moderiert }\end{array}$ \\
\hline $\begin{array}{l}\text { H19: Der Einfluss der } \\
\text { wahrgenommenen } \\
\text { Unterhaltung des Brand } \\
\text { Contents auf das emotionale } \\
\text { Markenimage ist stärker für } \\
\text { funktionale (im Vergleich } \\
\text { zu emotionalen) Marken }\end{array}$ & $\begin{array}{l}\text { H25: Der Einfluss der } \\
\text { wahrgenommenen } \\
\text { Unterhaltung des Brand } \\
\text { Contents auf das emotionale } \\
\text { Markenimage ist stärker für } \\
\text { Low-Involvement-Marken } \\
\text { im Vergleich zu } \\
\text { High-Involvement-Marken }\end{array}$ & $\begin{array}{l}\text { H19_N: Der Einfluss der } \\
\text { wahrgenommenen } \\
\text { Unterhaltung des Brand } \\
\text { Contents auf das emotionale } \\
\text { Markenimage wird durch die } \\
\text { Marke moderiert }\end{array}$ \\
\hline
\end{tabular}


Tabelle 7.22 (Fortsetzung)

\begin{tabular}{|c|c|c|}
\hline $\begin{array}{l}\text { Hypothese zur } \\
\text { Markenpositionierung (alt) }\end{array}$ & $\begin{array}{l}\text { Hypothese zum } \\
\text { Produktinvolvement (alt) }\end{array}$ & $\begin{array}{l}\text { Hypothese zu den Marken } \\
\text { (neu) }\end{array}$ \\
\hline $\begin{array}{l}\text { H20: Die Einstellung } \\
\text { gegenüber dem Brand } \\
\text { Content (a) und das } \\
\text { Globalimage der Marke (b) } \\
\text { sind höher, wenn der Brand } \\
\text { Content einer funktionalen } \\
\text { Marke in einem } \\
\text { informativen (anstatt einem } \\
\text { unterhaltenden) Kanal } \\
\text { gezeigt wird }\end{array}$ & $\begin{array}{l}\text { H26: Die Einstellung } \\
\text { gegenüber dem Brand } \\
\text { Content (a) und das } \\
\text { Globalimage der Marke (b) } \\
\text { sind höher, wenn der Brand } \\
\text { Content einer } \\
\text { High-Involvement-Marke in } \\
\text { einem informativen anstatt } \\
\text { einem unterhaltenden Kanal } \\
\text { gezeigt wird }\end{array}$ & \multirow[t]{2}{*}{$\begin{array}{l}\text { H20_N: Es gibt einen } \\
\text { Interaktionseffekt zwischen } \\
\text { Marke und Kanal betreffend } \\
\text { die Einstellung gegenüber } \\
\text { dem Brand Content (a) und } \\
\text { das Globalimage (b) }\end{array}$} \\
\hline $\begin{array}{l}\text { H21: Die Einstellung } \\
\text { gegenüber dem Brand } \\
\text { Content (a) und das } \\
\text { Globalimage der Marke (b) } \\
\text { sind höher, wenn der Brand } \\
\text { Content einer emotionalen } \\
\text { Marke in einem } \\
\text { unterhaltenden (anstatt } \\
\text { einem informativen) Kanal } \\
\text { gezeigt wird }\end{array}$ & $\begin{array}{l}\text { H27: Die Einstellung } \\
\text { gegenüber dem Brand } \\
\text { Content (a) und das } \\
\text { Globalimage der Marke (b) } \\
\text { sind höher, wenn der Brand } \\
\text { Content einer } \\
\text { Low-Involvement-Marke in } \\
\text { einem unterhaltenden anstatt } \\
\text { einem informativen Kanal } \\
\text { gezeigt wird }\end{array}$ & \\
\hline
\end{tabular}

Anmerkungen. Getestet werden im weiteren Verlauf die neuen Hypothesen in der rechten Spalte.

Zur Prüfung dieser Hypothesen wurde der Datensatz je nach Manipulation von Marke und Brand Content in acht Subsamples unterteilt (K.-J. Chen et al., 2015; Dahlén et al., 2008):

- Sample 1 (EL_IBC) mit $n=173$ : Elmex und informativer Brand Content

- Sample 2 (EL_UBC) mit $n=172$ : Elmex und unterhaltender Brand Content

- Sample 3 (RI_IBC) mit $n=173$ : Rivella und informativer Brand Content

- Sample 4 (RI_UBC) mit $n=171$ : Rivella und unterhaltender Brand Content

- Sample 5 (FR_IBC) mit $n=171$ : Freitag und informativer Brand Content

- Sample 6 (FR_UBC) mit $n=171$ : Freitag und unterhaltender Brand Content

- Sample 7 (VZ_IBC) mit $n=180$ : V-Zug und informativer Brand Content

- Sample 8 (VZ_UBC) mit $n=163$ : V-Zug und unterhaltender Brand Content 
Zuerst wurde eine CFA mit allen latenten Variablen des BCBI-Modells gerechnet (Gesamt-CFA). Die Analyse zeigte, dass Invarianz erfüllt ist (siehe elektronisches Zusatzmaterial Tabelle B-15). Es wurde zusätzlich ein Modell auf Invarianz gerechnet, welches nur die beiden abhängigen Variablen «Einstellung gegenüber dem Brand Content» und «Globalimage der Marke» beinhaltete. Hier wurde allerdings knapp keine Messinvarianz erreicht (siehe elektronisches Zusatzmaterial Tabelle B-17). Deshalb wurde in einem nächsten Schritt auf partielle Invarianz geprüft (Cieciuch \& Davidov, 2016). Dazu wurden die Faktorladungen und die Achsenabschnitte dreier Indikatoren ${ }^{8}$ frei geschätzt über die Gruppen. Damit konnte im reduzierten Modell, das nur die abhängigen Variablen beinhaltet, partielle Skalar-Invarianz hergestellt werden, was den Vergleich der Mittelwerte zulässt. Auf Basis partieller Skalar-Invarianz und der Gesamt-CFA wurden die folgenden Mittelwerte berechnet.

Die Einstellung gegenüber dem Brand Content ist bei Elmex signifikant höher beim informativen im Vergleich zu unterhaltendem Brand Content $\left(M_{I B C}-M_{U B C}\right.$ $=0.706, p<0.001$ ). Das Globalimage der Marke ist nicht signifikant höher $\left(M_{I B C}-M_{U B C}=0.051, p=0.613\right)$, wenn informativer Brand Content verwendet wird.

Bei der Marke Rivella unterscheiden sich weder die Einstellung gegenüber dem Brand Content $\left(M_{I B C}-M_{U B C}=0.235, p=0.060\right)$ noch das Globalimage $\left(M_{I B C}-M_{U B C}=-0.110, p=0.322\right)$ signifikant, je nachdem, ob informativer oder unterhaltender Brand Content präsentiert wurde. Dasselbe Resultat kann auch für Freitag ausgemacht werden. Die Einstellung gegenüber dem Brand Content $\left(M_{I B C}-M_{U B C}=0.099, p=0.390\right)$ und das Globalimage der Marke $\left(M_{I B C}-M_{U B C}=-0.017, p=0.889\right)$ werden nicht signifikant durch die Manipulation des Brand Contents beeinflusst. Bei V-Zug ist die Einstellung gegenüber dem Brand Content beim informativen Brand Content signifikant höher als beim unterhaltenden Brand Content $\left(M_{I B C}-M_{U B C}=0.447, p<0.001\right)$. Das Globalimage wird nicht signifikant unterschiedlich bewertet $\left(M_{I B C}-M_{U B C}=0.119, p\right.$ $=0.204)$.

Die Hypothesen lassen sich auch mit einem MIMIC-Vorgehen prüfen (Hancock, 2004). Dabei werden die totalen Effekte der Brand-Content-Manipulation auf die beiden abhängigen Variablen (Einstellung gegenüber Brand Content, Globalimage der Marke) berechnet. Um die totalen Effekte zu erhalten, wurde in Ergänzung zum BCBI-Modell ein direkter Pfad der Dummy-Variable der BrandContent-Manipulation auf die beiden abhängigen Variablen ergänzt. Weil weiter unten in gleicher Weise Effekte der Kanalmanipulation geprüft werden sollen,

\footnotetext{
${ }^{8}$ BC_ES_3, BC_ES_4 und M_GI_3.
} 
wurde ebenfalls ein direkter Pfad der Kanalmanipulation auf die beiden abhängigen Variablen «Einstellung gegenüber dem Brand Content» und «Globalimage der Marke» hinzugefügt. Dieses ergänzte Modell verfügte über einen guten Fit (Hair et al., 2014, S. 584): $d f=2^{\prime} 248, \chi^{2}=3903.441, p<0.001, \chi^{2} / d f=1.74$, $\mathrm{CFI}=0.955$, TLI $=0.952$, RMSEA $=0.046$ mit $90 \%$ K.I. zwischen 0.044 und 0.049, SRMR $=0.077$. Die totalen Effekte der Manipulation des Brand Contents. $(0=$ informativer Brand Content, $1=$ unterhaltender Brand Content $)$ auf die beiden abhängigen Variablen wurden in Mplus mittels Bootstrapping (5'000 Samples) $)^{9}$ berechnet.

Tabelle 7.23 Totale Effekte der Brand-Content-Manipulation nach Marken (MIMICVerfahren)

\begin{tabular}{l|l|l|l|r|r|l|l|l}
\hline AB & & UV & Marke & Stdy & Unstd & SE & \multicolumn{1}{l}{ C. R } & \multicolumn{1}{l}{$l$} \\
\hline BC_ES & $\leftarrow$ & BC_MP & Elmex & -0.602 & -0.616 & 0.111 & -5.551 & $0.000^{* * *}$ \\
\hline M_GI & $\leftarrow$ & BC_MP & Elmex & -0.123 & -0.106 & 0.088 & -1.203 & 0.229 \\
\hline BC_ES & $\leftarrow$ & BC_MP & Rivella & -0.290 & -0.313 & 0.121 & -2.588 & $0.009^{* *}$ \\
\hline M_GI & $\leftarrow$ & BC_MP & Rivella & 0.004 & 0.004 & 0.088 & 0.045 & 0.964 \\
\hline BC_ES & $\leftarrow$ & BC_MP & Freitag & -0.106 & -0.110 & 0.115 & -0.954 & 0.340 \\
\hline M_GI & $\leftarrow$ & BC_MP & Freitag & 0.021 & 0.022 & 0.105 & 0.213 & 0.831 \\
\hline BC_ES & $\leftarrow$ & BC_MP & V-Zug & -0.400 & -0.438 & 0.123 & -3.572 & $0.000^{* * *}$ \\
\hline M_GI & $\leftarrow$ & BC_MP & V-Zug & -0.159 & -0.128 & 0.082 & -1.560 & 0.112 \\
\hline
\end{tabular}

Anmerkungen. $\mathrm{AB}=$ Abhängige Variable. $\mathrm{UV}=$ Unabhängige Variable. BC_ES = Einstellung gegenüber Brand Content. M_GI = Globalimage der Marke. BC_MP = Manipulation des Brand Contents mit $0=$ informativ und $1=$ unterhaltend. Stdy $=$ Totaler standardisierter Effekt gemäss STDY-Standardisierung. Unstd = totaler unstandardisierter Effekt. SE = Standardfehler. C. R. = Critical Ratio. $p$-Wert auf Basis zweiseitiger Test. Resultate auf Basis einer Multigruppenanalyse. $* p<0.05, * * p<0.01, * * * p<0.001$.

Mit einer Ausnahme können hier die Resultate von oben bestätigt werden (siehe Tabelle. 7.23). Einzig der Effekt der Brand-Content-Manipulation auf die Einstellung gegenüber dem Brand Content bei der Marke Rivella erreicht bei MIMIC statistische Signifikanz, während beim Vorgehen nach Structured Means Modeling der Unterschied als nicht signifikant ausgewiesen wurde.

Zusammenfassend lässt sich sagen, dass die Einstellung gegenüber dem Brand Content bei mindestens zwei von vier Marken signifikant stärker ist beim informativen Brand Content. Die beiden Marken verfügen über eine funktionale

${ }^{9}$ Bei Bootstrapping wurde der ML-Schätzer verwendet (Muthén \& Muthén, 2017, S. 688). 
Markenpositionierung und über ein mittleres (Elmex) bzw. hohes (V-Zug) Produktinvolvement. Hinsichtlich des Globalimages können bei den Marken keine signifikanten Unterschiede ausgemacht werden, je nachdem, ob informativer oder unterhaltender Brand Content eingesetzt wird. Hypothese 16_N kann deshalb nur teilweise bestätigt werden (siehe Tabelle 7.24).

Tabelle 7.24 Test der Hypothese 16_N

\begin{tabular}{l|l|l|l}
\hline $\mathrm{Nr}$ & Hypothese & Koeffizienten & Beurteilung \\
\hline$H 16 \_N$ & $\begin{array}{l}\text { Es gibt einen Interaktionseffekt zwischen Marke } \\
\text { und Brand Content betreffend die Einstellung } \\
\text { gegenüber dem Brand Content (a) und das } \\
\text { Globalimage (b) }\end{array}$ & siehe Text & $\begin{array}{l}\text { teilweise } \\
\text { bestätigt }\end{array}$ \\
\hline
\end{tabular}

Die beiden nächsten Hypothesen besagen, dass der Einfluss der Informationsgratifikation des Brand Contents auf das funktionale Markenimage und der Einfluss der Unterhaltungsgratifikation des Brand Contents auf das emotionale Markenimage durch die Marke moderiert werden. Um die beiden Hypothesen zu testen, wurde das BCBI-Modell für alle vier Marken separat ausgewiesen mittels einer Multigruppenanalyse. Weil metrische Invarianz zwischen den Marken vorliegt, gemäss Prüfung eingangs des Kapitels (siehe elektronisches Zusatzmaterial Tabelle B-13), können die Parameter miteinander verglichen werden (Cieciuch \& Davidov, 2016).

Bei Gruppenvergleichen müssen unstandardisierte Koeffizienten $\beta_{\mathrm{u}}$ verglichen werden (Cieciuch \& Davidov, 2016). Der Einfluss der wahrgenommenen Information des Brand Contents auf das funktionale Markenimage beträgt für Elmex $\beta_{\mathrm{u}}=0.110(p=0.001)$, für Rivella. $\beta_{\mathrm{u}}=0.081(p=0.063)$, für Freitag $\beta_{\mathrm{u}}$ $=0.125(p=0.043)$ und für V-Zug $\beta_{\mathrm{u}}=0.161(p<0.001)$. Für die einzelnen Paarungen wurde jeweils überprüft, ob die Unterschiede zwischen den Marken signifikant sind. Der Berechnung des $p$-Wertes liegt ein $\chi^{2}$-Differenz-Test zugrunde (Muthén \& Muthén, 2017). Es konnten keine signifikanten Unterschiede festgestellt werden (siehe Tabelle 7.25).

Dies lässt für die hier vorliegenden Daten entgegen Hypothese 18_N den Schluss zu, dass der Effekt der wahrgenommenen Information auf das funktionale Markenimage unabhängig von der verwendeten Marke gleich ausfällt (siehe Tabelle 7.26).

Der Einfluss der wahrgenommenen Unterhaltung des Brand Contents auf das emotionale Markenimage beträgt für Elmex $\beta_{\mathrm{u}}=0.344(p<0.001)$, für Rivella $\beta_{\mathrm{u}}=0.250(p=0.002)$, für Freitag $\beta_{\mathrm{u}}=0.339(p<0.001)$ und für V-Zug $\beta_{\mathrm{u}}$ 
Tabelle 7.25 Test auf $\beta$-Unterschiede zwischen Marken: Information auf funktionales Markenimage

\begin{tabular}{l|l|l|l|l|l}
\hline Marke A & $\beta_{\text {uMarkeA }}$ & Marke B & $\beta_{\text {uMarkeB }}$ & Differenz & $p$ \\
\hline Elmex & 0.110 & Rivella & 0.081 & 0.028 & 0.604 \\
\hline Elmex & 0.110 & Freitag & 0.125 & -0.016 & 0.824 \\
\hline Elmex & 0.110 & V-Zug & 0.161 & -0.051 & 0.355 \\
\hline Rivella & 0.081 & Freitag & 0.125 & -0.044 & 0.559 \\
\hline Rivella & 0.081 & V-Zug & 0.161 & -0.08 & 0.202 \\
\hline Freitag & 0.125 & V-Zug & 0.161 & -0.036 & 0.639 \\
\hline
\end{tabular}

Anmerkungen. $\beta_{\mathrm{u}}=$ unstandardisierte Pfadkoeffizienten auf Basis einer Multigruppenanalyse.

Tabelle 7.26 Test der Hypothese 18_N

\begin{tabular}{l|l|l|l}
\hline $\mathrm{Nr}$ & Hypothese & Koeffizienten & Beurteilung \\
\hline$H 18 \_N$ & $\begin{array}{l}\text { Der Einfluss der wahrgenommenen Information des } \\
\text { Brand Contents auf das funktionale Markenimage } \\
\text { wird durch die Marke moderiert }\end{array}$ & siehe Text & abgelehnt \\
\hline
\end{tabular}

$=0.175(p=0.004)$. Der Effekt der wahrgenommenen Unterhaltung auf das emotionale Markenimage wird ebenfalls nicht durch die Marke moderiert (siehe Tabelle 7.27).

Tabelle 7.27 Test auf $\beta$-Unterschiede zwischen Marken: Unterhaltung auf emotionales Markenimage

\begin{tabular}{l|l|l|l|c|l}
\hline Marke A & $\beta_{\text {uMarkeA }}$ & Marke B & $\beta_{\text {uMarkeB }}$ & Differenz & $p$ \\
\hline Elmex & 0.344 & Rivella & 0.250 & 0.094 & 0.360 \\
\hline Elmex & 0.344 & Freitag & 0.339 & 0.005 & 0.964 \\
\hline Elmex & 0.344 & V-Zug & 0.175 & 0.169 & 0.053 \\
\hline Rivella & 0.250 & Freitag & 0.339 & -0.089 & 0.452 \\
\hline Rivella & 0.250 & V-Zug & 0.175 & 0.075 & 0.450 \\
\hline Freitag & 0.339 & V-Zug & 0.175 & 0.164 & 0.123 \\
\hline
\end{tabular}

Anmerkungen. $\beta_{\mathrm{u}}=$ unstandardisierte Pfadkoeffizienten auf Basis einer Multigruppenanalyse.

Entsprechend findet sich in dieser Studie auch keine Bestätigung für Hypothese 19_N (siehe Tabelle 7.28. 
Tabelle 7.28 Test der Hypothese 19_N

\begin{tabular}{l|l|l|l}
\hline $\mathrm{Nr}$ & Hypothese & Koeffizienten & Beurteilung \\
\hline$H 19 \_N$ & $\begin{array}{l}\text { Der Einfluss der wahrgenommenen Unterhaltung } \\
\text { des Brand Contents auf das emotionale } \\
\text { Markenimage wird durch die Marke moderiert }\end{array}$ & siehe Text & abgelehnt \\
\hline
\end{tabular}

Schliesslich wird überprüft, ob eine Marke-Kanal-Interaktion die Einstellung gegenüber dem Brand Content und das Globalimage beeinflusst. Der Datensatz wurde wiederum in acht Gruppen aufgeteilt (K.-J. Chen et al., 2015; Dahlén et al., 2008; Ryffel et al., 2014):

- Sample 1 (EL_TW) mit $n=169$ : Elmex und Twitter

- Sample 2 (EL_IN) mit $n=176$ : Elmex und Instagram

- Sample 3 (RI_TW) mit $n=165$ : Rivella und Twitter

- Sample 4 (RI_IN) mit $n=179$ : Rivella und Instagram

- Sample 5 (FR_TW) mit $n=169$ : Freitag und Twitter

- Sample 6 (FR_IN) mit $n=173$ : Freitag und Instagram

- Sample 7 (VZ_TW) mit $n=172$ : V-Zug und Twitter

- Sample 8 (VZ_IN) mit $n=171:$ V-Zug und Instagram

Die acht Gruppen wurden für Vergleichszwecke auf Skalar-Invarianz überprüft mittels einer CFA über alle Variablen des BCBI-Modells (Cieciuch \& Davidov, 2016; Hair et al., 2017). Es zeigte sich, dass Skalar-Invarianz über alle Gruppen vorliegt (siehe elektronisches Zusatzmaterial Tabelle B-19). In einem zweiten Schritt wurde wiederum ein reduziertes Modell gerechnet, welches Invarianz nur bei den beiden abhängigen Variablen «Einstellung gegenüber dem Brand Content» und «Globalimage der Marke» prüft. Auch hier konnte Messinvarianz aufgezeigt werden (siehe elektronisches Zusatzmaterial Tabelle B-21). Die folgenden Mittelwerte basieren auf den Berechnungen für die Gesamt-CFA.

Bei Elmex können keine signifikanten Unterschiede in Bezug auf die Einstellung gegenüber dem Brand Content festgestellt werden, je nachdem, ob der Brand Content auf Twitter oder auf Instagram ausgespielt wird $\left(M_{T W}-M_{I N}=-0.077\right.$, $p=0.502)$. Das Globalimage der Marke wird hingegen signifikant besser beurteilt, wenn der Brand Content auf Instagram präsentiert wurde $\left(M_{T W}-M_{I N}=-\right.$ 0.324, $p=0.001$ ). Bei Rivella werden - wird der Brand Content auf Instagram ausgespielt - weder die Einstellung gegenüber dem Brand Content $\left(M_{T W}-M_{I N}\right.$ $=-0.072, p=0.559)$ noch das Globalimage $\left(M_{T W}-M_{I N}=-0.076, p=0.493\right)$ 
signifikant besser beurteilt. Auch bei Freitag können keine signifikanten Unterschiede auf die Einstellung gegenüber dem Brand Content $\left(M_{T W}-M_{I N}=0.070\right.$, $p=0.527)$ oder auf das Globalimage $\left(M_{T W}-M_{I N}=-0.009, p=0.939\right)$ identifiziert werden, je nachdem, ob der Brand Content auf Twitter oder Instagram ausgespielt wurde. Dasselbe gilt auch für V-Zug. Weder die Einstellung gegenüber dem Brand Content $\left(M_{T W}-M_{I N}=0.147, p=0.223\right)$ noch das Globalimage $\left(M_{T W}-M_{I N}=-0.013, p=0.888\right)$ werden durch die Manipulation des Kanals signifikant beeinflusst.

Analog zur Hypothese 16_N wurden auch hier die Effekte zusätzlich über das MIMIC-Vorgehen geprüft (Hancock, 2004). Die totalen Effekte der Kanalmanipulation auf die beiden abhängigen Variablen «Einstellung gegenüber dem Brand Content» und «Globalimage der Marke» wurden mittels Bootstrapping (5000 Samples) und ML-Schätzung berechnet (siehe Tabelle 7.29).

Tabelle 7.29 Totale Effekte der Kanalmanipulation nach Marken (MIMIC-Verfahren)

\begin{tabular}{l|l|l|l|r|r|l|r|l}
\hline AB & & UV & Marke & Stdy & Unstd & \multicolumn{1}{l}{ SE } & \multicolumn{1}{l}{ C. R } & \multicolumn{1}{l}{$p$} \\
\hline BC_ES & $\leftarrow$ & K_MP & Elmex & 0.045 & 0.046 & 0.058 & 0.790 & 0.429 \\
\hline M_GI & $\leftarrow$ & K_MP & Elmex & 0.207 & 0.177 & 0.073 & 2.420 & $0.012^{*}$ \\
\hline BC_ES & $\leftarrow$ & K_MP & Rivella & 0.068 & 0.074 & 0.057 & 1.289 & 0.197 \\
\hline M_GI & $\leftarrow$ & K_MP & Rivella & 0.096 & 0.087 & 0.076 & 1.146 & 0.252 \\
\hline BC_ES & $\leftarrow$ & K_MP & Freitag & -0.037 & -0.038 & 0.053 & -0.713 & 0.476 \\
\hline M_GI & $\leftarrow$ & K_MP & Freitag & 0.090 & 0.098 & 0.081 & 1.206 & 0.228 \\
\hline BC_ES & $\leftarrow$ & K_MP & V-Zug & 0.005 & 0.006 & 0.060 & 0.094 & 0.925 \\
\hline M_GI & $\leftarrow$ & K_MP & V-Zug & 0.011 & 0.009 & 0.061 & 0.142 & 0.887 \\
\hline
\end{tabular}

Anmerkungen. $\mathrm{AB}=$ Abhängige Variable. $\mathrm{UV}=$ Unabhängige Variable. $\mathrm{BC} \_\mathrm{ES}=$ Einstellung gegenüber Brand Content. M_GI = Globalimage der Marke. K_MP = Manipulation des Kanals mit $0=$ informativer Kanal (Twitter) und $1=$ unterhaltender Kanal (Instagram). Stdy $=$ Totaler standardisierter Effekt gemäss STDY-Standardisierung. Unstd = totaler unstandardisierter Effekt. $\mathrm{SE}=$ Standardfehler. C. $\mathrm{R} .=$ Critical Ratio. $p$-Wert auf Basis zweiseitiger Test. Resultate auf Basis einer Multigruppenanalyse. ${ }^{*} p<0.05$.

Die mittels Structured Means Modeling oben gefundenen Resultate bezüglich Signifikanz können durch MIMIC bestätigt werden. Zusammenfassend lässt sich sagen, dass die Einstellung gegenüber dem Brand Content bei allen vier Marken unabhängig vom verwendeten Kanal ist. Das Globalimage der Marke hängt nur bei Elmex vom Kommunikationskanal ab und wird in Instagram signifikant besser beurteilt. Es können dementsprechend entgegen Hypothese 20_N 
keine Interaktionseffekte zwischen Kanal und Marke festgestellt werden (siehe Tabelle 7.30

Tabelle 7.30 Test der Hypothese 20

\begin{tabular}{l|l|l|l}
\hline $\mathrm{Nr}$ & Hypothese & Koeffizienten & Beurteilung \\
\hline$H 20 \_N$ & $\begin{array}{l}\text { Es gibt einen Interaktionseffekt zwischen Marke } \\
\text { und Kanal betreffend die Einstellung gegenüber } \\
\text { dem Brand Content (a) und das } \\
\text { Globalimage (b) }\end{array}$ & siehe Text & abgelehnt \\
\hline
\end{tabular}

\subsection{Zusätzliche Post-hoc-Analysen}

In diesem Kapitel wird der Einfluss von Kontrollvariablen betrachtet. Zudem werden zusätzliche Effekte durch kategoriale Moderatoren ausgewiesen.

\subsubsection{Kontrollvariablen}

Kontrollvariablen spielen bei Experimenten insbesondere dann eine Rolle, wenn der Zuteilung der Teilnehmenden auf die Experimentalgruppen keine perfekte Randomisierung zugrunde liegt (Hancock, 2004). Im Rahmen dieser Studie konnte die Zuteilung zufällig erfolgen. Dementsprechend sollten die Kontrollvariablen den Einfluss der Manipulation auf die «konzeptuellen unabhängigen Variablen» (MacKenzie, 2001) bzw. Mediatoren Informations- und Unterhaltungsgratifikation des Brand Contents sowie Informations- und Unterhaltungserwartungen des Kanals nicht beeinflussen. Auch die Signifikanz der weiteren Pfadkoeffizienten sollte sich durch die Integration der Pfadkoeffizienten nicht ändern. Insgesamt wurden sechs Kontrollvariablen geprüft: Alter, Geschlecht, Bildung, Einkommen, Social-Media-Affinität und Wiederkauf. Die Kontrollvariablen wurden jeweils als Prädikatoren in das BCBI-Modell integriert (Hancock, 2004, S. 325). Alle latenten Variablen wurden auf die jeweils eingesetzte Kontrollvariable regrediert. Die Modelle verfügten allesamt über eine hohe Güte (siehe elektronisches Zusatzmaterial Tabelle B-23; Hair et al., 2014, S. 584).

Die erhaltene Signifikanz der Pfadkoeffizienten des BCBI-Modells (siehe Abbildung 7.1) ging durch die Integration der Kontrollvariablen nicht verloren. Einzig der Einfluss der Einstellung gegenüber dem Kanal auf das Globalimage der Marke $(\beta=-0.04, p=0.021$ ohne Kontrollvariablen) verliert durch die 
Kontrollvariablen «Social-Media-Affinität» $(\beta=0.017, p=0.354)$ und «Wiederkauf» $(\beta=-0.031, p=0.059)$ seine Signifikanz. Allerdings wurde bereits oben dargelegt, dass der besagte Effekt trotz Signifikanz im BCBI-Modell aufgrund seiner Stärke als irrelevant angesehen werden muss (Chin, 1998).

Die Kontrollvariablen beeinflussen allerdings gewisse abhängige Variablen über alle Experimentalsituationen hinweg (siehe elektronisches Zusatzmaterial Tabelle B-24). Die Informations- $(\beta=-0.321, p<0.001)$ und Unterhaltungserwartungen $(\beta=-0.573, p<0.001)$ gegenüber dem Kommunikationskanal sind in der Altersgruppe «40-65 Jahre» signifikant tiefer als in der Altersgruppe «18-39 Jahre». Die Social-Media-Affinität (SMA) beeinflusst die Informations$(\beta=0.479, p<0.001)$ und Unterhaltungserwartungen $(\beta=0.448, p<0.001)$ gegenüber dem Kanal positiv. Das Geschlecht hat einen Einfluss auf die Unterhaltungserwartungen gegenüber dem Kanal. Diese fallen bei Männern $(\beta=-$ $0.260, p<0.001)$ tiefer aus.

Mit steigender SMA steigen die wahrgenommene Information $(\beta=0.161$, $p<0.001)$ und die wahrgenommene Unterhaltung $(\beta=0.205, p<0.001)$ des Brand Contents. Personen mit höherer Bildung schätzen die wahrgenommene Information des Brand Contents tiefer ein. $(\beta=-0.108, p=0.025)$ und auch das Einkommen wirkt sich negativ auf die wahrgenommene Information $(\beta=-$ $0.154, p=0.014)$ und auf die wahrgenommene Unterhaltung $(\beta=-0.174, p=$ 0.013) des Brand Contents aus.

Personen, welche die ihnen gezeigte Marke bereits gekauft haben und diese wieder kaufen würden, schätzen sowohl das funktionale $(\beta=0.595, p<0.001)$ als auch das emotionale Markenimage $(\beta=0.6205, p<0.001)$ und das Globalimage der Marke $(\beta=0.356, p<0.001)$ höher ein als diejenigen Personen, welche die Marke noch nicht gekauft haben oder diese nicht mehr kaufen würden. In der Altersgruppe «40-65 Jahre» wird das funktionale Image höher beurteilt als in der Altersgruppe «18-39 Jahre» $(\beta=0.15, p=0.014)$. Auch Personen mit tertiärer Bildung bewerten das funktionale Markenimage höher $(\beta=0.113, p=0.044)$. Eine Kontrolle nach Geschlecht zeigt, dass Männer das emotionale Markenimage etwas tiefer einstufen als Frauen $(\beta=-0.121, p=0.024)$. SMA wirkt sich schliesslich positiv auf das markenbezogene Online-Verhalten aus $(\beta=0.144, p$ $<0.001)$. Personen mit hoher SMA würden entsprechend eher die Webseite der beworbenen Marke besuchen und den Brand Content in sozialen Medien teilen.

In einer weiteren Variable wurde erhoben, ob der Brand Content zur Marke passt (Übereinstimmung). Die wahrgenommene Übereinstimmung ist beim informativen Brand Content. $(M=3.92, S D=1.044)$ signifikant höher als beim unterhaltenden Brand Content $(M=3.28, S D=1.249)$, wie ein t-Test mit unabhängigen Stichproben (Rasch, Friese, Hofmann \& Naumann, 2014a) zeigt ( $\mathrm{T}=$ 
9.817, $d f=1206.944, p<0.001, d=0.636,95 \% \mathrm{KI}$ für $d[0.509,0.763])$. Die Einstellung gegenüber dem Brand Content wird positiv durch die Übereinstimmung beeinflusst $(\beta=0.199, p<0.001)$, wenn die Variable im BCBI-Modell integriert wird.

Mittels einer einzelnen Variable wurde die Ex-ante-Einstellung gegenüber den vier Marken erhoben. Für alle vier Marken wurde das BCBI-Modell inklusive der jeweiligen Kontrollvariable separat ausgewiesen. Dabei wurden die latenten Variablen funktionales Markenimage, emotionales Markenimage sowie Globalimage auf die Kontrollvariable regrediert. Die Güte der vier markenbezogenen Modelle war gut, einzig der SRMR-Wert war bei drei Marken über dem Schwellenwert (siehe elektronisches Zusatzmaterial Tabellen B-25 bis B-29). Es zeigte sich, dass das funktionale und das emotionale Markenimage bei allen vier Marken signifikant positiv durch die Ex-ante-Einstellung gegenüber der Marke beeinflusst werden. Die Effekte des emotionalen und funktionalen Markenimages auf das Globalimage bleiben jedoch auch unter Berücksichtigung der Ex-ante-Einstellung bestehen. Bei den Effekten von wahrgenommener Information und Unterhaltung auf das funktionale und emotionale Markenimage ergaben sich nur geringfügige Veränderungen. Drei der Pfade verloren durch die Integration der Kontrollvariable ihre signifikante Wirkung: der Effekt der Unterhaltung auf das funktionale Markenimage bei Elmex (ohne Kontrollvariable: $\beta=0.131, p=0.027$; mit Kontrollvariable: $\beta=0.065, p=0.293$ ) sowie die Effekte der Information auf das funktionale Markenimage (ohne Kontrollvariable: $\beta=0.179, p=0.006$; mit Kontrollvariable: $\beta=0.100, p=0.170$ ) und der Information auf das emotionale Markenimage (ohne Kontrollvariable: $\beta=0.146, p=0.048$; mit Kontrollvariable: $\beta=0.078, p=0.330$ ) bei Rivella. Der Effekt der wahrgenommenen Unterhaltung auf das emotionale Markenimage - der stärkste der vier Effekte bleibt auch unter Einbezug der Kontrollvariable Ex-ante-Einstellung bei allen vier Marken hochsignifikant.

\subsubsection{Zusätzliche Moderationsanalysen}

Für die zusätzlichen Moderationsanalysen wurde die Stichprobe jeweils in zwei Gruppen aufgeteilt, auf Invarianz geprüft und das BCBI-Modell für beide Gruppen mittels Multigruppenanalyse separat gerechnet (Hancock, 2004). Anhand eines $\chi^{2}$-Differenz-Tests wurde überprüft, ob sich die Pfadkoeffizienten zwischen den Gruppen unterscheiden (Muthén \& Muthén, 2017). Alle signifikanten Effekte und die Invarianztests sind im elektronischen Zusatzmaterial (siehe Tabellen B-30 bis B-39) aufgelistet. Hier werden die wesentlichen Resultate wiedergegeben. 
Eingangs wurde dargelegt, dass die Manipulation des Kanals nicht vollumfänglich erfolgreich war. Zwar werden mit Instagram höhere Unterhaltungserwartungen verbunden, bezüglich der Informationserwartungen unterscheiden sich Instagram und Twitter allerdings nicht signifikant. Wird die Stichprobe allerdings in zwei Altersgruppen aufgeteilt (18-39 Jahre, $n=805$; 40-65 Jahre, $n=567$ ), dann zeigt sich, dass die Manipulation für die jüngere Stichprobe funktioniert. Die Teilnehmenden zwischen 18 und 39 Jahren haben signifikant höhere Unterhaltungserwartungen $\left(\beta_{\mathrm{u}}=0.263, p=0.003\right)$ und signifikant tiefere Informationserwartungen $\left(\beta_{\mathrm{u}}=-0.315, p=0.001\right)$ gegenüber Instagram.

Der Einfluss der Unterhaltungserwartungen gegenüber dem Kanal auf die Einstellung gegenüber dem Kanal ist bei Frauen $\left(\beta_{\mathrm{u}}=0.635, p<0.001 ; n=708\right)$ signifikant höher als bei Männern $\left(\beta_{\mathrm{u}}=0.327, p<0.001 ; n=666\right)$ und bei 18- bis 39-Jährigen $\left(\beta_{\mathrm{u}}=0.596, p<0.001\right)$ signifikant grösser als bei 40 - bis 65-Jährigen $\left(\beta_{\mathrm{u}}=0.238, p=0.056\right)$.

Die Manipulation des Brand Contents $(0=$ informativ, $1=$ unterhaltend $)$ wirkt sich bei den 18- bis 39-Jährigen stärker auf die wahrgenommene Information des Brand Contents. $\left(\beta_{\mathrm{u}}=-1.277, p<0.001\right)$ aus als bei den 40 - bis 65 -Jährigen $\left(\beta_{\mathrm{u}}=-0.970, p<0.001\right)$.

Open Access Dieses Kapitel wird unter der Creative Commons Namensnennung 4.0 International Lizenz (http://creativecommons.org/licenses/by/4.0/deed.de) veröffentlicht, welche die Nutzung, Vervielfältigung, Bearbeitung, Verbreitung und Wiedergabe in jeglichem Medium und Format erlaubt, sofern Sie den/die ursprünglichen Autor(en) und die Quelle ordnungsgemäß nennen, einen Link zur Creative Commons Lizenz beifügen und angeben, ob Änderungen vorgenommen wurden.

Die in diesem Kapitel enthaltenen Bilder und sonstiges Drittmaterial unterliegen ebenfalls der genannten Creative Commons Lizenz, sofern sich aus der Abbildungslegende nichts anderes ergibt. Sofern das betreffende Material nicht unter der genannten Creative Commons Lizenz steht und die betreffende Handlung nicht nach gesetzlichen Vorschriften erlaubt ist, ist für die oben aufgeführten Weiterverwendungen des Materials die Einwilligung des jeweiligen Rechteinhabers einzuholen.

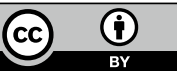

